\title{
Evaluation of the Microvascular Research Center Training Program for Assessing Microsurgical Skills in Trainee Surgeons
}

\author{
Seiji Komatsu ${ }^{1}$, Kiyoshi Yamada ${ }^{1}$, Shuji Yamashita ${ }^{2}$, Narushi Sugiyama ${ }^{1}$, Eijiro Tokuyama ${ }^{1}$, \\ Kumiko Matsumoto ${ }^{1}$, Ayumi Takara ${ }^{1}$, Yoshihiro Kimata ${ }^{1}$ \\ ${ }^{1}$ Department of Plastic and Reconstructive Surgery, Okayama University Hospital, Okayama; ${ }^{2}$ Department of Plastic and Reconstructive \\ Surgery, Okayama Saiseikai General Hospital, Okayama, Japan
}

Background We established the Microvascular Research Center Training Program (MRCP) to help trainee surgeons acquire and develop microsurgical skills. Medical students were recruited to undergo the MRCP to assess the effectiveness of the MRCP for trainee surgeons. Methods Twenty-two medical students with no prior microsurgical experience, who completed the course from 2005 to 2012, were included. The MRCP comprises 5 stages of training, each with specific passing requirements. Stages 1 and 2 involve anastomosing silicone tubes and blood vessels of chicken carcasses, respectively, within 20 minutes. Stage 3 involves anastomosing the femoral artery and vein of live rats with a 1-day patency rate of $>80 \%$. Stage 4 requires replantation of free superficial inferior epigastric artery flaps in rats with a 7-day success rate of $>80 \%$. Stage 5 involves successful completion of one case of rat replantation/ transplantation. We calculated the passing rate for each stage and recorded the number of anastomoses required to pass stages 3 and 4 .

Results The passing rates were 100\% (22/22) for stages 1 and 2, 86.4\% (19/22) for stage $3,59.1 \%(13 / 22)$ for stage 4 , and $55.0 \%(11 / 20)$ for stage 5 . The number of anastomoses performed was $17.2 \pm 12.2$ in stage 3 and $11.3 \pm 8.1$ in stage 4 .

Conclusions Majority of the medical students who undertook the MRCP acquired basic microsurgical skills. Thus, we conclude that the MRCP is an effective microsurgery training program for trainee surgeons.

Keywords Microsurgery / Program evaluation / Tissue transplantation
Correspondence: Seiji Komatsu Department of Plastic and Reconstructive Surgery, Okayama University Hospital, 2-5-1 Shikata-cho, Kita-ku, Okayama 700-8558, Japan Tel: +81-86-235-7214 Fax: +81-86-235-7210 E-mail: komats-s@cc.okayama-u.ac.jp
This article was presented at the inauguration meeting of the International Microsurgery Simulation Society, June 30, 2012 in London, UK.

This work was partially supported by a Grant-in-Aid for Young Scientists (B) 23972045 provided by the Ministry of Education, Culture, Sports, Sciences, and Technology of Japan.

No potential conflict of interest relevant to this article was reported.

Received: 26 Jan 2013 • Revised: 22 Feb 2013 • Accepted: 26 Feb 2013

pISSN: 2234-6163 • elSSN: 2234-6171 • http://dx.doi.org/10.5999/aps.2013.40.3.214 • Arch Plast Surg 2013;40:214-219

\section{INTRODUCTION}

Trainee surgeons rarely receive sufficient opportunities to acquire and develop their microsurgical skills. Although many trainee surgeons are considerably enthusiastic about learning microsurgery, they are unable to do so because of the lack of a comprehensive standardized microsurgery training program. Furthermore, criteria for assessing a trainee surgeon's microsurgical skills are inadequate. The Objective Structured Assessment of Technical Skills (OSATS) is a popular tool to evaluate surgical ability $[1,2]$. However, there is no standardized method to assess microsurgical skills [3-7]. Without appropriate assess- 


\section{Fig. 1. The Microvascular Research Center Training Program (MRCP)}

The MRCP comprises 5 stages. Acceptance criteria are set for each stage. Participating trainee surgeons are not permitted to anastomose human blood vessels until they pass stage 4. SIEA, superficial inferior epigastric artery.

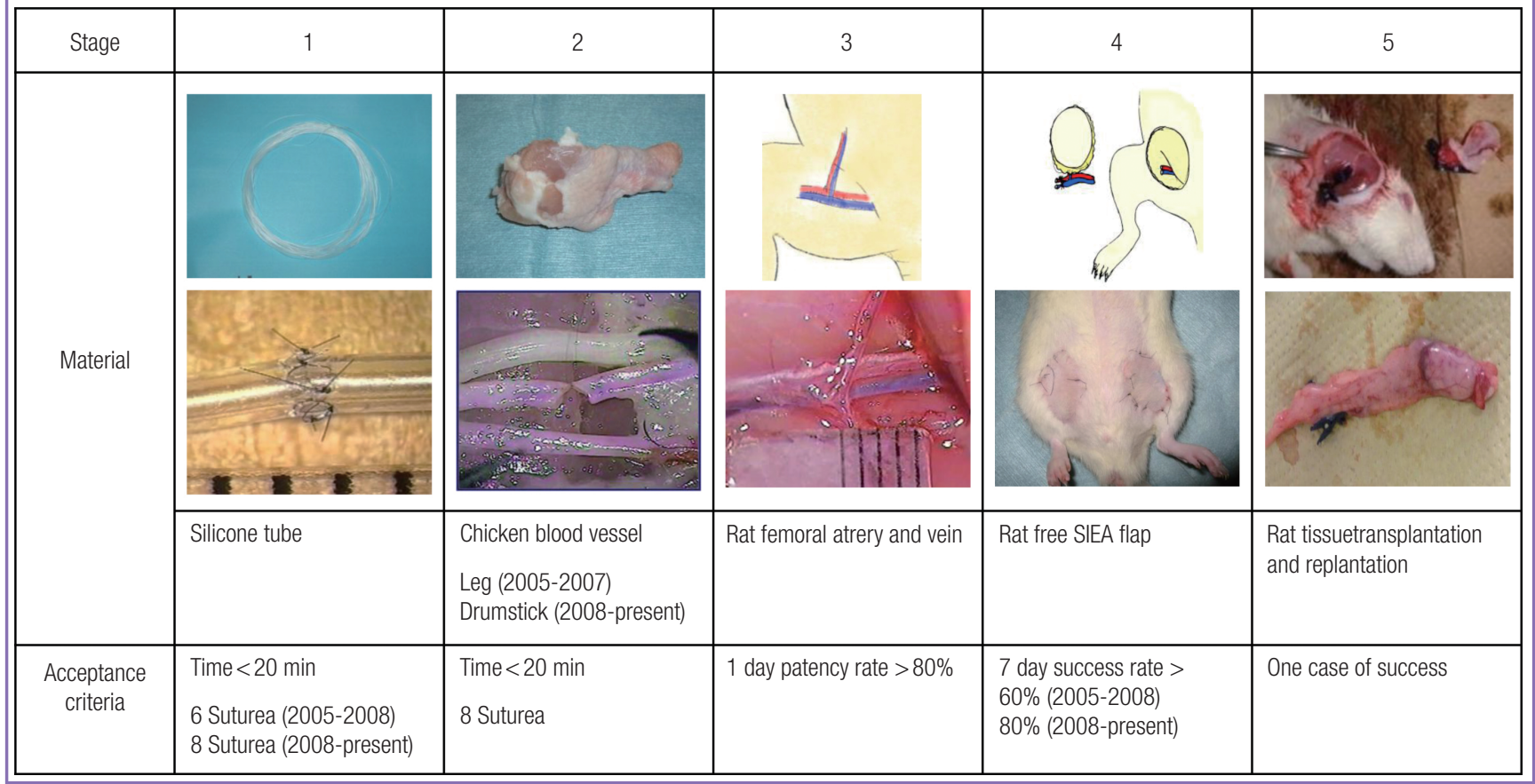

ment of trainees' microsurgical skills by experienced surgeons, novice surgeons may not be provided the opportunity to perform vascular anastomoses. Thus, establishment of a standardized microsurgery training program to assess trainee surgeons' skill is required.

To address this need, we established the Microvascular Research Center Training Program (MRCP) in 2005, comprising progressive skill level requirements (Fig. 1) to help trainee surgeons acquire microsurgical skills. Because there is no diploma in microsurgery currently available in Japan, our department established its own Diploma in Microsurgery and established the passing criteria for each stage. Trainee surgeons are not allowed to perform anastomoses of human blood vessels until they successfully complete the MRCP. The program is reviewed annually and often updated to maximize its effectiveness. We assessed the effectiveness of the MRCP by evaluating the results of medical students with no prior surgical experience.

\section{METHODS}

All rats used in the program were housed in a climate-controlled facility and were anesthetized with an intraperitoneal injection of $50 \mathrm{mg} / \mathrm{kg}$ pentobarbital sodium. Water and pellet food produced specifically for experimental animals were provided ad libitum. All animal experiments were performed at the Depart- ment of Animal Resources, Advanced Science Research Center, with the approval of our university's Animal Research Committee and were conducted in strict accordance with the National Institutes of Health guidelines.

As part of our university's Medical Research Internship, third year medical students were assigned to various departments to engage in research for 3 months. In the Department of Plastic and Reconstructive Surgery, they completed the MRCP for a 3-month period and participated in tissue transplantation experiments. From 2005 to 2012, 22 medical students were enrolled in this program. Although these students had knowledge of human anatomy, they had no prior surgical experience. Guidance was provided by board-certified plastic surgeons who completed the MRCP.

\section{Outline of the MRCP}

In stage 1 , trainees acquire basic microsurgical skills and anastomose silicone tubes measuring $1 \mathrm{~mm}$ in diameter using 10-0 nylon sutures. Silicone tubes are stiffer than blood vessels, thus making it easier to confirm lumen patency in silicone tubes.

In stage 2, trainees cut and anastomose the blood vessels of chicken carcasses using 10-0 nylon sutures. These vessels are more similar to human vessels than the silicone tubes used in stage 1 . Thus, stage 2 provides more practical training.

In stage 3 , trainees attempt to anastomose the femoral vessels 
Fig. 2. Case 1: face allotransplantation

$(A, B)$ The harvested hemiface flap from the dorsal and ventral surface. Anastomoses of the external carotid arteries and external jugular veins were performed. (C) Lateral views of the recipient on postoperative day 7.
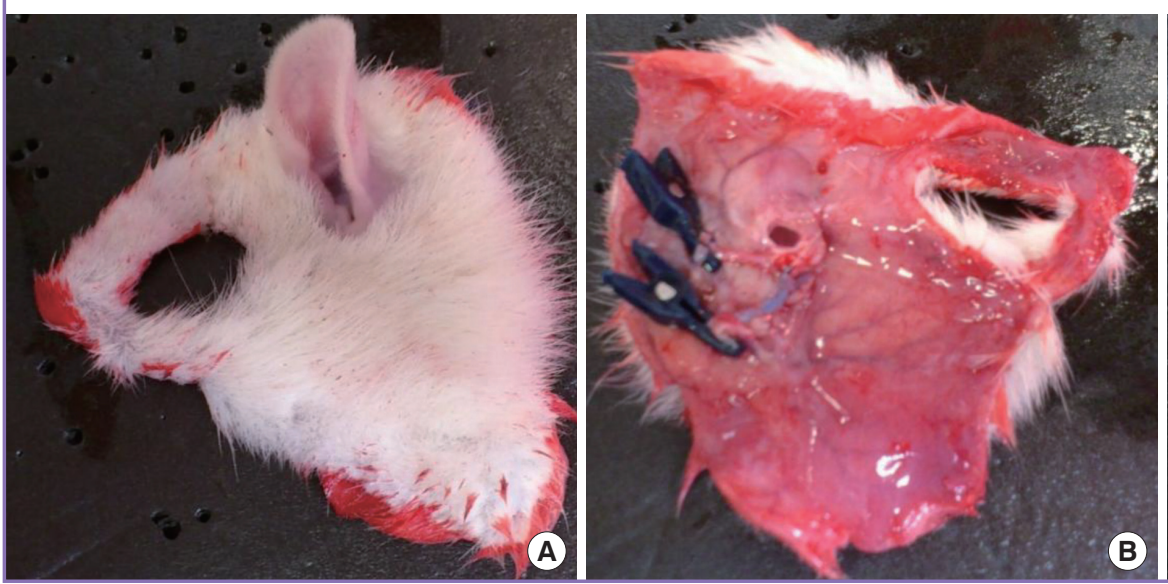

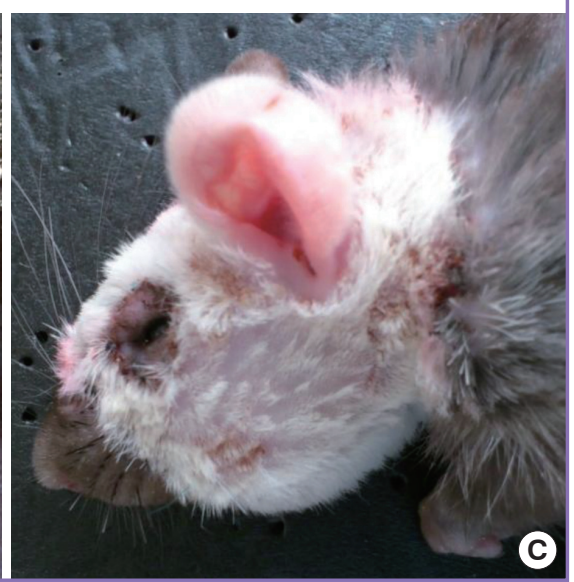

(approximately $1 \mathrm{~mm}$ in diameter) of live rats. This phase provides an opportunity to practice dissection and hemostasis and to perfect anastomotic techniques using live specimens.

In stage 4, trainees attempt to replant free flaps in rats using superficial inferior epigastric artery (SIEA) flaps [8]. Anastomoses of the femoral arteries and veins are required, thus allowing confirmation of long-term patency of these blood vessels. Trainee surgeons who participate in this course are not permitted to anastomose human blood vessels until they pass stage 4 .

In stage 5, trainees attempt rat tissue replantation/transplantation. In consultation with trainers, trainees select the procedures needed for the presenting case.

Trainers evaluate the trainees at each stage. Acceptable MRCP passing criteria are as follows: in stage 1 (Fig. 1), trainees are required to anastomose a silicone tube using eight sutures within 20 minutes. In stage 2, trainees are required to anastomose a blood vessel from the second leg segment of a chicken carcass using eight sutures within 20 minutes. In stage 3, the 1-day patency rate of anastomosed femoral arteries and veins of a rat should be $>80 \%$. In stage 4, the 7-day success rate of a free SIEA flap replantation in a rat, for which the last five cases are assessed, must be $>80 \%$. In stage 5 , trainees must successfully complete one advanced rat tissue replantation/transplantation operation.

We calculated the passing rate for each stage and recorded the number of anastomoses required to pass stages 3 and 4. Anastomosis of an artery and vein was counted as one.

\section{MRCP revisions}

Stage 5 of the MRCP was established in 2006 because we believed an advanced course was necessary to produce more competent surgeons. This revision was also intended to secure feedback from medical students for our animal experiments. In stage 5, rat tissue replantation and transplantation are attempted, requiring the use of microsurgical skills acquired in stage 4 . The trainees select the advanced procedures in stage 5 in consultation with the trainers.

In 2008, three criteria were also revised as follows: 1) In stage 1 , the number of required sutures was changed from six to eight because mastery of eight sutures was necessary for stage 2.2) In stage 2, the area of the chicken leg used for vessel anastomoses was changed to more appropriately match the diameter of the blood vessel used in stage 3.3) In stage 4, because the reported success rate for free flaps was $>90 \%$, the required success rate for flap replantation was increased from $60 \%$ to $80 \%$ to bridge the gap between the acceptance criterion and the actual success rate.

\section{Examples of procedures performed:}

\section{Case 1: Face allotransplantation}

This procedure was performed according to a paper by Demir et al. [9]. In allotransplantation, one Lewis rat served as the donor and one Lewis-Brown Norway rat served as the recipient (Fig. 2 ). Anastomoses of the external carotid arteries and external jugular veins were performed. Cyclosporine A was administered as an immunosuppressant.

\section{Case 2: Penis isotransplantation}

This procedure was performed according to a paper by Sonmez et al. [10]. Lewis rats served as both donor and recipient. The penis was transplanted to the recipient's right thigh to avoid anastomosis with the urethra. The donor's dorsal penile vein was anastomosed to the recipient's right femoral vein, and the donor's corpus spongiosum was anastomosed to the recipient's femoral artery (Fig. 3). Immunosuppressants were not adminis- 
Fig. 3. Case 2: penis isotransplantation

$(A, B)$ The harvested penile graft was transplanted to the recipient's right thigh. The donor's dorsal penile vein was anastomosed to the recipient's right femoral vein, and the donor's corpus spongiosum was anastomosed to the recipient's femoral artery.
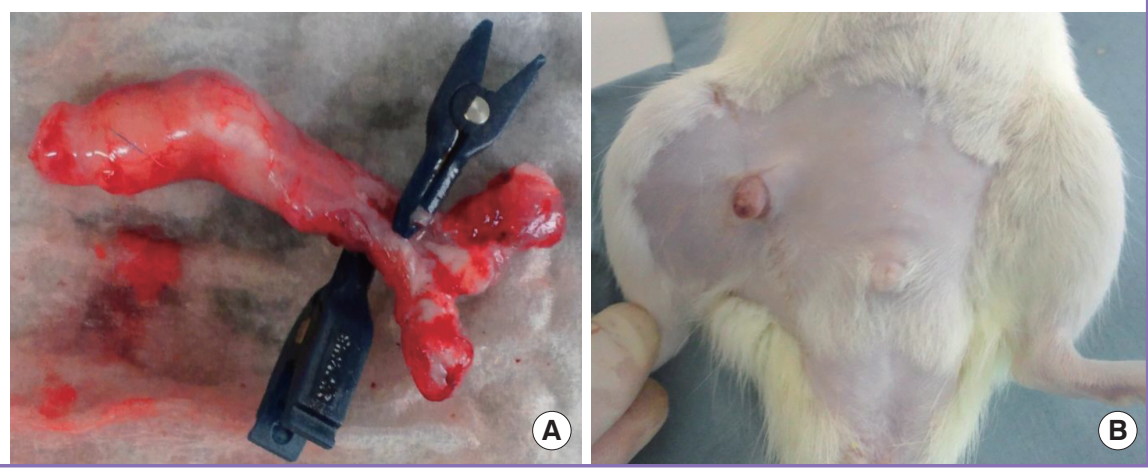

\section{Fig. 4. Student passing rate per stage}

The passing rate was 100\% (22/22) for stage $1,100 \%$ (22/22) for stage $2,86.4 \%(19 / 22)$ for stage $3,59.1 \%(13 / 22)$ for stage 4 , and $55.0 \%$ $(11 / 20)$ for stage 5 .

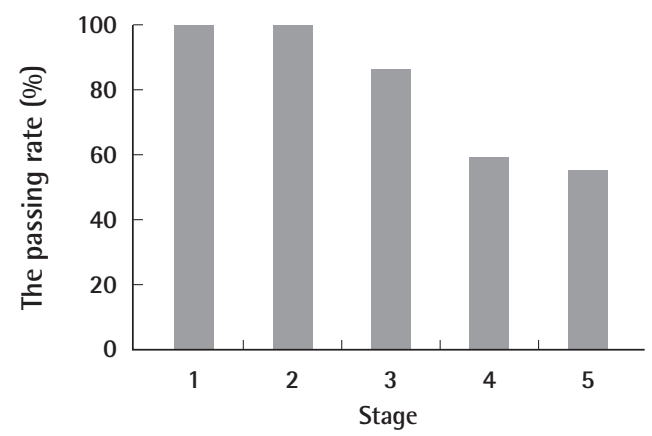

tered in this case.

\section{RESULTS}

The passing rate was $100 \%(22 / 22)$ for stage $1,100 \%(22 / 22)$ for stage $2,86.4 \%(19 / 22)$ for stage $3,59.1 \%(13 / 22)$ for stage 4 , and $55.0 \%(11 / 20)$ for stage 5 (Fig. 4$)$. The number of anastomoses performed was $17.2 \pm 12.2$ in stage 3 and $11.3 \pm 8.1$ in stage 4 . The following procedures were performed by medical students in stage 5 (Table 1): lymphatic vessel anastomosis $(2 / 2)$, free SIEA flap replantation with anastomoses of the superficial epigastric artery and vein, (1/1), face allotransplantation $(1 / 1)$, penis isotransplantation $(1 / 1)$, testis isotransplantation $(1 / 1)$, thymus isotransplantation $(1 / 1)$, forelimb replantation $(1 / 1)$, toe replantation $(1 / 1)$, ear replantation $(1 / 1)$, free latissimus dorsi flap replantation $(0 / 1)$, vascularized lymphatic vessel graft (1/1), and vascularized nerve graft (0/1).

The majority of medical students who participated in the MRCP passed stage 3 and acquired basic microsurgical skills despite having no prior microsurgical experience.
Table 1. Results of medical students who underwent the MRCP

\begin{tabular}{|c|c|c|c|c|c|}
\hline \multirow{3}{*}{$\begin{array}{c}\text { No. } \\
1\end{array}$} & \multirow{3}{*}{$\begin{array}{l}\text { Year } \\
2005\end{array}$} & \multirow{2}{*}{\multicolumn{2}{|c|}{$\begin{array}{c}\text { Stage } 3 \text { Stage } 4 \\
\text { No. of anasto- } \\
\text { moses needed } \\
\text { to pass }\end{array}$}} & \multicolumn{2}{|l|}{ Stage 5} \\
\hline & & & & Procedures & Results \\
\hline & & 43 & - & - & - \\
\hline 2 & 2005 & 38 & - & - & - \\
\hline 3 & 2006 & 22 & 5 & Thymus isotransplantation & Success \\
\hline 4 & 2006 & 18 & 5 & $\begin{array}{l}\text { Vascularized lymphatic vessel } \\
\text { graft }\end{array}$ & Success \\
\hline 5 & 2006 & 44 & 20 & Free latissimus dorsi flap & Failure \\
\hline 6 & 2007 & 13 & 5 & Toe replantation & Success \\
\hline 7 & 2007 & 15 & 5 & $\begin{array}{l}\text { Free SIEA flap replantation (SIEA } \\
\text { and SIEV were anastomosed) }\end{array}$ & Success \\
\hline 8 & 2007 & 10 & - & - & - \\
\hline 9 & 2008 & 8 & 7 & Vascularized nerve graft & Failure \\
\hline 10 & 2008 & 19 & - & - & - \\
\hline 11 & 2009 & 13 & 5 & Ear replantation & Success \\
\hline 12 & 2009 & - & - & - & - \\
\hline 13 & 2009 & - & - & & - \\
\hline 14 & 2010 & 8 & 8 & Testis isotransplantation & Success \\
\hline 15 & 2010 & 7 & - & - & - \\
\hline 16 & 2010 & - & - & - & - \\
\hline 17 & 2011 & 6 & 23 & Face allotransplantation & Success \\
\hline 18 & 2011 & 10 & 5 & Forelimb replantation & Success \\
\hline 19 & 2011 & 19 & 16 & Penis isotransplantation & Success \\
\hline 20 & 2012 & 10 & 28 & Lymphatic vessel anastomosis & Success \\
\hline 21 & 2012 & 7 & 15 & Lymphatic vessel anastomosis & Success \\
\hline 22 & 2012 & - & - & - & - \\
\hline
\end{tabular}

\section{DISCUSSION}

On the basis of the results presented, we consider the MRCP to be an effective microsurgery training program for trainee surgeons. The passing rate in stage 4 was $59.1 \%$, and the principal cause of failure was delayed venous thrombosis. We attributed the lower passing percentage to specific surgical problems such as the use of bipolar coagulators near blood vessels, unneces- 
sary contact with the intima of blood vessels, and inappropriate suture spacing. Incidence of venous thrombi could be reduced by addressing these problems. Of the 13 medical students who passed stage 4, 11 (84.6\%) also passed stage 5. The biggest obstacle was between stages 3 and 4 . Once the medical students acquired the necessary skills to pass stage 4, it was not as difficult to perform tissue transplantation and replantation; therefore, we can conclude that passing stage 4 is a reasonable acceptance criterion for trainee surgeons.

Feedback from the medical students was useful while reviewing the MRCP. As described previously, many improvements were made on the basis of their opinions. In 2007, responding to a perceived need, we distributed an instructional video on flap elevation and blood vessel anastomosis. In 2010, we uploaded this video on YouTube to provide free access to our trainees and other aspiring medical professionals worldwide [11]. Although the criteria were made more stringent in 2008 , the passing rate continues to improve; this may be attributed to the increased effectiveness of the MRCP due to ongoing revisions. In 2011, we established a training manual and distributed an atlas of rat anatomy to trainees at their request.

We plan to make further improvements to the program. Objective scoring of microsurgical skills is necessary. The surgeons assessing the trainees' skills may be somewhat subjective; therefore, we wish to establish clearer criteria. The OSATS is a popular, objective, and validated tool, but it is not possible to assess detailed microsurgical skills using this instrument $[1,2]$. Several objective and validated methods for assessing microsurgical skills have recently been reviewed [3-7]. These methods should be incorporated into the MRCP to record objective scores. The use of animals for experiments should also be reduced. Many alternatives for learning microsurgery have been reported [12-21], but it is impossible to assess blood vessel leakage and thrombosis in inanimate models [22-24]. Although we use silicone tubes and blood vessels from chicken carcasses in the initial stages, it is very difficult to eliminate the use of live animals completely.

In this study, we described the results of medical students who underwent the MRCP; however, the MRCP is intended for trainee surgeons. In our hospital, trainee surgeons who have completed the MRCP perform microsurgeries such as free flaps, finger replantations, and lymphatic venous anastomoses with a success rate of $>90 \%$. Furthermore, the MRCP improves their skills and increases their motivation.

We believe that increasing the number of trainee surgeons skilled in microsurgery will raise the overall quality of plastic surgery. To bring this plan to fruition, the MRCP is offered to physicians worldwide. We have enrolled eight young plastic surgeons in their 20's and 30's from other hospitals in Japan and two from overseas. Since 2011, we have been accepting applications from plastic surgeons in Myanmar as part of our Myanmar-Japan Plastic and Reconstructive Surgery Educational Project. We believe that the MRCP could contribute toward the global development of plastic surgery. Thus we have continued to revise our MRCP in the hope that it will become the global standard for microsurgery training.

\section{REFERENCES}

1. Martin JA, Regehr G, Reznick R, et al. Objective structured assessment of technical skill (OSATS) for surgical residents. Br J Surg 1997;84:273-8.

2. Nugent E, Joyce C, Perez-Abadia G, et al. Factors influencing microsurgical skill acquisition during a dedicated training course. Microsurgery 2012;32:649-56.

3. Selber JC, Chang EI, Liu J, et al. Tracking the learning curve in microsurgical skill acquisition. Plast Reconstr Surg 2012; 130:551e-8e.

4. Balasundaram I, Aggarwal R, Darzi LA. Development of a training curriculum for microsurgery. Br J Oral Maxillofac Surg 2010;48:598-606.

5. Temple CL, Ross DC. A new, validated instrument to evaluate competency in microsurgery: the University of Western Ontario Microsurgical Skills Acquisition/Assessment instrument. Plast Reconstr Surg 2011;127:215-22.

6. Chan W, Niranjan N, Ramakrishnan V. Structured assessment of microsurgery skills in the clinical setting. J Plast Reconstr Aesthet Surg 2010;63:1329-34.

7. Chan WY, Matteucci P, Southern SJ. Validation of microsurgical models in microsurgery training and competence: a review. Microsurgery 2007;27:494-9.

8. Yamashita S, Sugiyama N, Hasegawa K, et al. A novel model for supermicrosurgery training: the superficial inferior epigastric artery flap in rats. J Reconstr Microsurg 2008;24:53743.

9. Demir Y, Ozmen S, Klimczak A, et al. Tolerance induction in composite facial allograft transplantation in the rat model. Plast Reconstr Surg 2004;114:1790-801.

10. Sonmez E, Nasir S, Siemionow M. Penis allotransplantation model in the rat. Ann Plast Surg 2009;62:304-10.

11. Okayama University Department of Plastic and Reconstructive Surgery [Internet]. San Bruno, CA: YouTube, LLC; c2013 [cited 2013 Mar 13]. Available from: http://www. youtube.com/user/okayamauniversityprs.

12. Lannon DA, Atkins JA, Butler PE. Non-vital, prosthetic, and virtual reality models of microsurgical training. Microsurgery 2001;21:389-93. 
13. Crosby NL, Clapson JB, Buncke HJ, et al. Advanced nonanimal microsurgical exercises. Microsurgery 1995;16:6558.

14. Klein I, Steger U, Timmermann W, et al. Microsurgical training course for clinicians and scientists at a German University hospital: a 10-year experience. Microsurgery 2003; 23:461-5.

15. Furka I, Brath E, Nemeth N, et al. Learning microsurgical suturing and knotting techniques: comparative data. Microsurgery 2006;26:4-7.

16. Peled IJ, Kaplan HY, Wexler MR. Microsilicone anastomoses. Ann Plast Surg 1983;10:331-2.

17. Govila A. A simple model on which to practise microsurgical technique: a fresh chicken. Br J Plast Surg 1981;34:486-7.

18. Hino A. Training in microvascular surgery using a chicken wing artery. Neurosurgery 2003;52:1495-7.

19. Martins PN, Montero EF. Basic microsurgery training: comments and proposal. Acta Cir Bras 2007;22:79-81.

20. Lahiri A, Lim AY, Qifen Z, et al. Microsurgical skills training: a new concept for simulation of vessel-wall suturing. Microsurgery 2005;25:21-4.

21. Uson J, Calles MC. Design of a new suture practice card for microsurgical training. Microsurgery 2002;22:324-8.

22. Akyurek M, Safak T, Oztekin C, et al. Dorsal penile vein as a new training model for microvenous anastomosis in rats. Ann Plast Surg 2002;49:280-4.

23. Dunn RM, Mancoll J. Flap models in the rat: a review and reappraisal. Plast Reconstr Surg 1992;90:319-28.

24. Zhang F, Sones WD, Lineaweaver WC. Microsurgical flap models in the rat. J Reconstr Microsurg 2001;17:211-21. 